Article

\title{
Lack of Correlation between Facial Sexual Dimorphism, Fluctuating Asymmetry and Self-Perceived Attractiveness in Men and Women
}

\author{
Stefan Van Dongen ${ }^{1, *\left(\mathbb{D}, \text { Omid Ekrami }^{1}(\mathbb{D}) \text { and Peter Claes }\right.}{ }^{2,3,4} \mathbb{( D}$ \\ 1 Evolutionary Ecology Group, Biology Department, Antwerp University, 2610 Wilrijk, Belgium; \\ omid.ekrami@uantwerpen.be \\ 2 Medical Imaging Research Center, Universitair Ziekenhuis Gasthuisberg, 3000 Leuven, Belgium \\ 3 Department of Electrical Engineering, ESAT/PSI, 3001 Heverlee, Belgium \\ 4 Department of Human Genetics, KU Leuven, 3000 Leuven, Belgium; peter.claes@kuleuven.be \\ * Correspondence: Stefan.vandongen@uantwerpen.be; Tel.: +32-32653336
}

Received: 11 December 2019; Accepted: 31 January 2020; Published: 4 February 2020

\begin{abstract}
Human morphological sexual dimorphism and fluctuating asymmetry have been suggested to signal 'individual quality' and are therefore expected to covary as well as to correlate with surrogate fitness/quality measures like attractiveness and dominance. While some case studies indeed found support for these hypotheses, the overall evidence is not overwhelming. However, most previous research used a limited number of landmarks to quantify masculinity and asymmetry. We here present results based on high-density 3D scans covering the entire facial area. In spite of these more detailed measurements, no significant associations were detected. These results are in line with conclusions of recent meta-analyses and cast doubt on the role of masculinity and fluctuating asymmetry as signals of (genetic) quality.
\end{abstract}

Keywords: fluctuating asymmetry; masculinity; developmental instability; sexual dimorphism; genetic quality

\section{Introduction}

The condition-dependent male ornamentation theory predicts that signaling traits of individual phenotypical and/or genetic quality can only develop under favorable conditions. In humans, two such candidate signaling traits have been put forward, namely morphological sexual dimorphism and fluctuating asymmetry. For decades, fluctuating asymmetry (FA, random deviations from perfect symmetry) has been considered as a measure of developmental instability (DI, the sensitivity of an organisms' development to developmental perturbations [1]) and has been studied in an evolutionary context in many species, including humans. FA may reflect stress, poor health and/or low 'genetic quality'. However, as shown in a meta-analysis of the human literature [2] overall associations are often weak, albeit being overall statistically significant. The role of morphological asymmetries has also been argued to be important with respect to attractiveness (as a proxy for mating success and fitness), where evidence for the evolutionary relevance has also been argued to be ambiguous [3]. Next to FA, human morphological masculinity has repeatedly been put forward as a signal of individual 'quality'. The immunocompetence handicap hypothesis is presumed to play a key role, where individuals exposed to higher androgen levels can withstand their immunodepression and afford to develop more masculine traits [4]. It consequently has been hypothesized that masculinity in men (and to some extent femininity in women) and symmetry-another presumed costly condition-dependent trait-would reflect correlated signals of quality, which some case studies support [5,6]. Nevertheless, a meta-analysis of the literature did not find strong evidence for this hypothesis [7], and a more recent 
study in primate skulls did not find any support either [8]. Furthermore, the absence of a link between human facial masculinity and genetic diversity of the MHC has challenged the immunocompetence handicap hypothesis as well [4].

In conclusion, evidence for morphological measures of masculinity and developmental instability to (jointly) signal quality are ambiguous at best. However, weak associations between FA and other covariates are not unexpected, because FA in single traits only weakly reflects the underlying process of DI, herewith biasing associations downward [9]. To overcome this problem, it is useful to base measurements on as many independent traits as possible. In this paper, we use high-density 3D facial scans in humans to measure both facial FA and masculinity, based on over 7000 vertices. Obviously, different points/vertices on a face often do not represent independent developmental events, but nevertheless, in this way a better and more complete analysis of the entire face is possible. In doing so, the most powerful analysis with highest expected effect sizes can be expected. Next to estimating this association, we also relate them to two presumed fitness traits; namely, attractiveness and handgrip strength. Individual attractiveness has been linked to several quality measures including mating success, physical fitness and health. In this study, self-perceived attractiveness, a proxy of how others rate one's attractiveness [10], is studied. In addition, self-perceived attractiveness is also influenced by other factors, like self-esteem and how others react towards yourself. In that way, it may reflect a more integrated general measure of attractiveness, including objective and subjective external and internal drivers, which have never been studied in relation to FA before. There is growing evidence that handgrip strength (HGS) can be considered as a general measure of health, physical fitness, psychological well-being, dominance and reproductive success [11]. Furthermore, self-perceived attractiveness and HGS also appear to correlate positively [12]. We explore two major hypotheses using high-density 3D facial scans. Our main goal is to test for associations between facial masculinity and FA in men and women. In men, more masculine faces are expected to be more symmetric, while in women, less masculine (i.e., more feminine) faces are predicted to be more symmetric [6]. Second, we study to what extent facial masculinity and FA correlate with self-perceived attractiveness and HGS.

\section{Materials and Methods}

In November 2018, students from the University of Antwerp were invited by email to participate in this study. In total, 74 males and 93 females participated. In this range of sample sizes, often applied in this area of research [7], a correlation coefficient of 0.3 can be detected statistically with a power of $80 \%$. All individuals were informed about the study procedure and signed an informed consent document. Data collection and handling was approved by the ethics commission of the University of Antwerp (SHW_18_03). All participants completed a short questionnaire to determine, age, sex, height and weight, after which hand strength was measured using a hand dynamometer for both hands. Next, a 3D surface scan of the face was obtained for all participants using a handheld structured-light scanner (Artec ${ }^{\mathrm{TM}}$ Eva, Artec Group, Luxembourg). The participants were instructed to keep a neutral face during the scan.

For the analyses of the 3D scans and to obtain facial shape and facial asymmetry measures, we followed Ekrami et al. [13]. In short, a completely symmetric spatially dense anthropometric mask with paired vertices was non-rigidly mapped onto the target faces using an Iterative Closest Point registration algorithm. The average facial asymmetry (in $\mathrm{mm}$ ) was calculated for males and females separately, after correction for mean asymmetry (i.e., directional asymmetry) [13]. A score for the degree of masculinity of the face was obtained after partial least squares (PLS) analysis of the facial shape coordinates. PLS analysis tries to find a vector in space that maximizes the correlation between the coordinates of all vertices and sex, resulting in a hyperplane maximizing the sexual dimorphism. The male (mean $=0.29, \mathrm{SD}=0.28$ ) and female (mean $=-0.24, \mathrm{SD}=0.22$ ) scores differed significantly $\left(t_{160}=13.2, p<0.0001\right)$, but showed substantial overlap in distribution. The associations between masculinity, facial asymmetry, HGS and self-perceived attractiveness were based on Pearson correlation coefficients in men and women separately. 


\section{Results}

In neither men nor women, facial masculinity showed significant correlations with facial FA (men: $\mathrm{r}=0.17$ [95\% C.I.: -0.08-0.37], $p=0.15$; women: $\mathrm{r}=-0.06$ (95\% C.I.: $-0.23-0.18$ ], $p=0.60$, Figure 1). While facial masculinity did correlate significantly with HGS (men: $r=0.27$ [95\% C.I.: 0.05-0.47], $p=0.02$; women: $\mathrm{r}=0.30$ [95\% C.I.: 0.08-0.51], $p=0.01$ ), it did not correlate significantly with self-perceived attractiveness (men: $\mathrm{r}=0.06$ [95\% C.I.: $-0.18-0.23$ ], $p=0.62$; women: $\mathrm{r}=0.12$ [95\% C.I.: $-0.06-0.30], p=0.24$, Figure 2). Finally, facial asymmetry did not correlate significantly with self-perceived attractiveness (men: $\mathrm{r}=-0.10$ [95\% C.I.: $-0.29-0.07$ ], $p=0.39$; women: $\mathrm{r}=-0.00$ [95\% C.I.: $-0.19-0.18], p=0.96$, Figure 2).

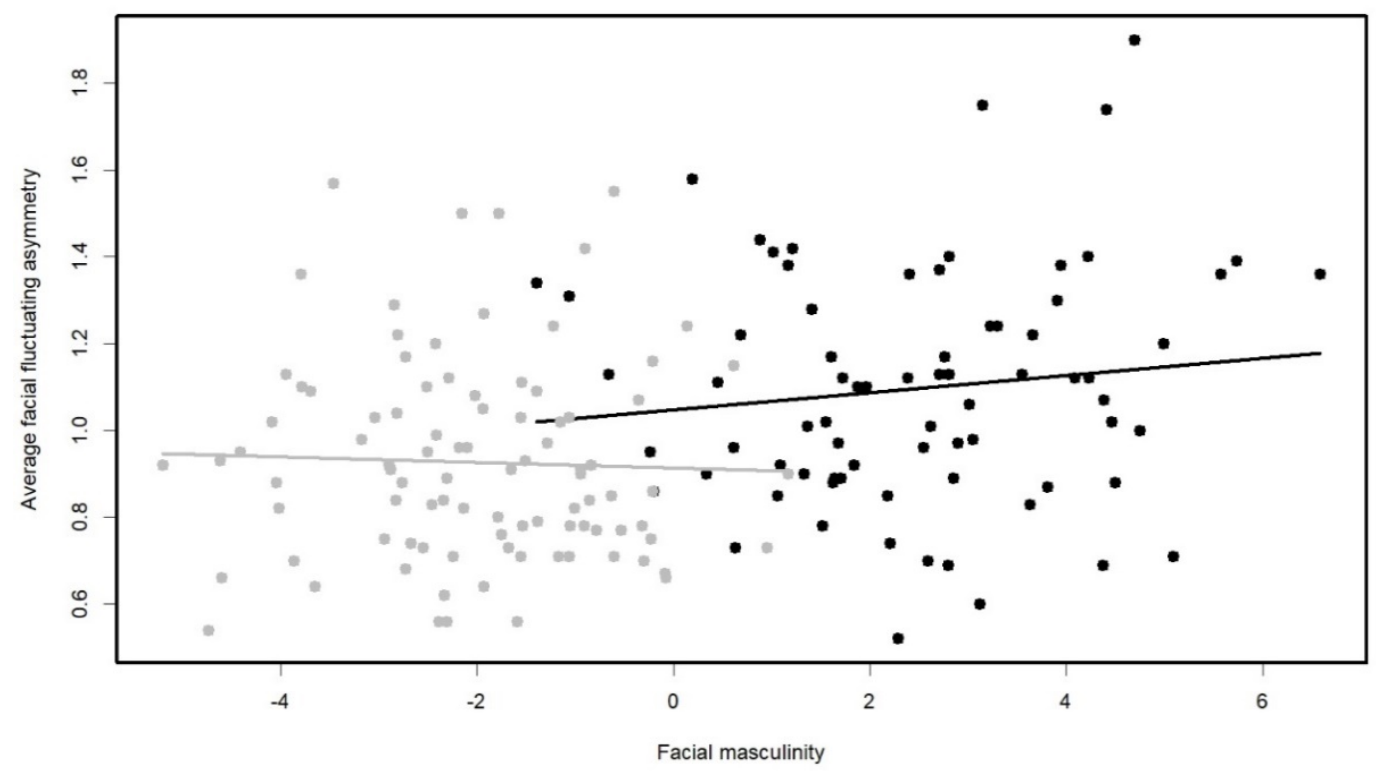

Figure 1. Observed associations between facial masculinity and average facial fluctuating asymmetry in men (black) and women (grey). Estimated regression lines were added to illustrate the sign of the association, but slopes did not differ significantly from zero (see text for details).
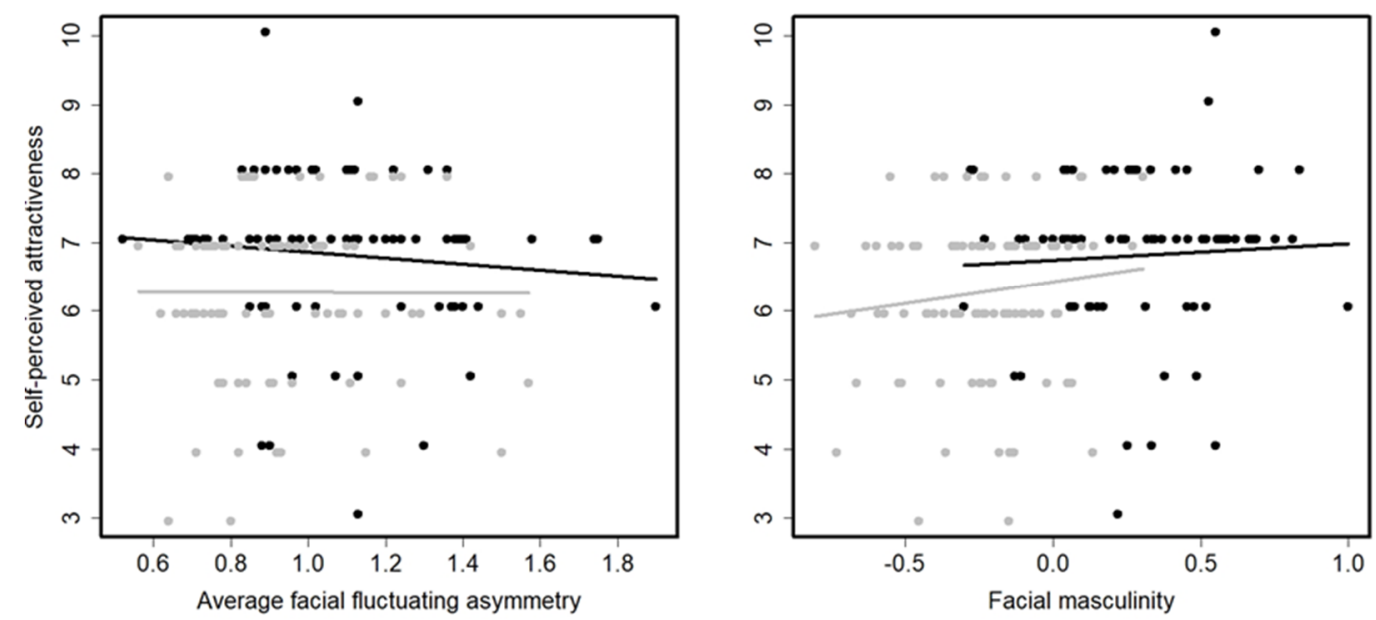

Figure 2. Observed associations between self-perceived attractiveness and both facial masculinity and average facial fluctuating asymmetry in men (black) and women (grey). Estimated regression lines were added to illustrate the sign of the association, but slopes did not differ significantly from zero (see text for details). 


\section{Discussion and Conclusions}

This study does not support evidence that facial symmetry and masculinity are correlated and reflect two traits that are biologically linked during development as claimed before [6,7]. While both masculinity and asymmetry were based on a very large number of quasi-landmarks, capturing basically all morphological variations, the correlations were even in the opposite direction to that expected a priori. We thus conclude that there is little evidence that sexual dimorphism and fluctuating asymmetry should be considered as two interrelated signaling traits. In addition, facial FA did not correlate with self-perceived attractiveness and HGS, our two measures of presumed individual quality. Facial masculinity did correlate positively with HGS in a comparable way in both men and women, which is likely driven by the effects of androgen exposure on muscle development.

Author Contributions: Conceptualization, S.V.D., O.E. and P.C.; methodology, O.E.; formal analysis, O.E.; writing-original draft preparation, S.V.D.; writing-review and editing, S.V.D., O.E. and P.C. All authors have read and agree to the published version of the manuscript.

Funding: This research received no external funding.

Conflicts of Interest: The authors declare no conflict of interest.

\section{References}

1. Klingenberg, C.P. A Developmental Perspective on Developmental Instability: Theory, Models, and Mechanisms. In Developmental Instability: Causes and Consequences; Oxford University Press: Oxford, UK, 2007; Volume 3, pp. 154-196.

2. Van Dongen, S.; Gangestad, S.W. Human fluctuating asymmetry in relation to health and quality: A meta-analysis. Evol. Hum. Behav. 2011, 32, 380-398. [CrossRef]

3. Van Dongen, S. Associations between asymmetry and human attractiveness: Possible direct effects of asymmetry and signatures of publication bias. Ann. Hum. Biol. 2011, 38, 317-323. [CrossRef] [PubMed]

4. Zaidi, A.A.; White, J.D.; Mattern, B.C.; Liebowitz, C.R.; Puts, D.A.; Claes, P.; Shriver, M.D. Facial Masculinity Does Not Appear to Be a Condition-Dependent Male Ornament and Does Not Reflect MHC Heterozygosity in Humans. Proc. Natl. Acad. Sci. USA 2019, 116, 1633-1638. [CrossRef] [PubMed]

5. Brown, W.M.; Price, M.E.; Kang, J.S.; Pound, N.; Zhou, Y.; Yu, H. Fluctuating asymmetry and preferences for sex-typical bodily characteristics. Proc. Natl. Acad. Sci. USA 2008, 105, 12938-12943. [CrossRef] [PubMed]

6. Little, A.C.; Jones, B.C.; Waitt, C.; Tiddeman, B.P.; Feinberg, D.R.; Perrett, D.I.; Apicella, C.L.; Marlowe, F.W. Symmetry is related to sexual dimorphism in faces: Data across culture and species. PLoS ONE 2008, 3, e2106. [CrossRef]

7. Van Dongen, S. Fluctuating asymmetry and masculinity/femininity in humans: A meta-analysis. Arch. Sex. Behav. 2012, 41, 1453-1460. [CrossRef]

8. Van Dongen, S. Lack of correlation between fluctuating asymmetry and morphological masculinity/femininity in primate skulls. Int. J. Primatol. 2015, 36, 113-123. [CrossRef]

9. Whitlock, M. The heritability of fluctuating asymmetry and the genetic control of developmental stability. Proc. R. Soc. Lond. Ser. B 1996, 263, 849-854.

10. Muñoz-Reyes, J.A.; Fernández, A.M.; Flores-Prado, L.; Guerra, R.; Turiégano, E. Fighting ability influences mate value in late adolescent men. Pers. Individ. Dif. 2015, 80, 46-50. [CrossRef]

11. Gallup, A.C.; Fink, B. Handgrip Strength as a Darwinian Fitness Indicator in Men. Front. Psychol. 2018, 9, 439. [CrossRef] [PubMed]

12. Sneade, M.; Furnham, A. Hand grip strength and self-perceptions of physical attractiveness and psychological well-being. Evol. Psychol. Sci. 2016, 2, 123-128. [CrossRef]

13. Ekrami, O.; Claes, P.; White, J.D.; Zaidi, A.A.; Shriver, M.D.; Van Dongen, S. Measuring asymmetry from high-density 3D surface scans: An application to human faces. PLoS ONE 2018, 13, e0207895. [CrossRef] [PubMed] 